\title{
Differentiating Metastatic and Non-metastatic Tumor Cells from Their Translocation Profile through Solid-State Micropores
}

Waqas Ali ${ }^{1,2,3}$, Azhar llyas ${ }^{1,2,3}$, Loan Bui ${ }^{4}$, Bailey Sayles ${ }^{4}$, Yeun Hur ${ }^{4}$, Young-Tae $\mathrm{Kim}^{3,4,5}$ and Samir M. Iqbal ${ }^{1,2,3,4,5, *}$

${ }^{1}$ Nano-Bio Lab, ${ }^{2}$ Department of Electrical Engineering, ${ }^{3}$ Nanotechnology

Research Center, ${ }^{4}$ Department of Bioengineering, University of Texas at Arlington, Arlington, Texas 76019. ${ }^{5}$ Department of Urology, University of Texas Southwestern Medical Center at Dallas, Dallas, Texas 75235.

*Contact Author:

Samir M. lqbal, Ph.D., P.E., FRSC

Associate Professor

Department of Electrical Engineering

University of Texas at Arlington

500 S. Cooper St \#217

Arlington, Texas 76019

United States of America

Email: SMIQBAL@uta.edu

Ph: $\quad+1-817-272-0228$

Fax: $\quad+1-817-272-7458$ 


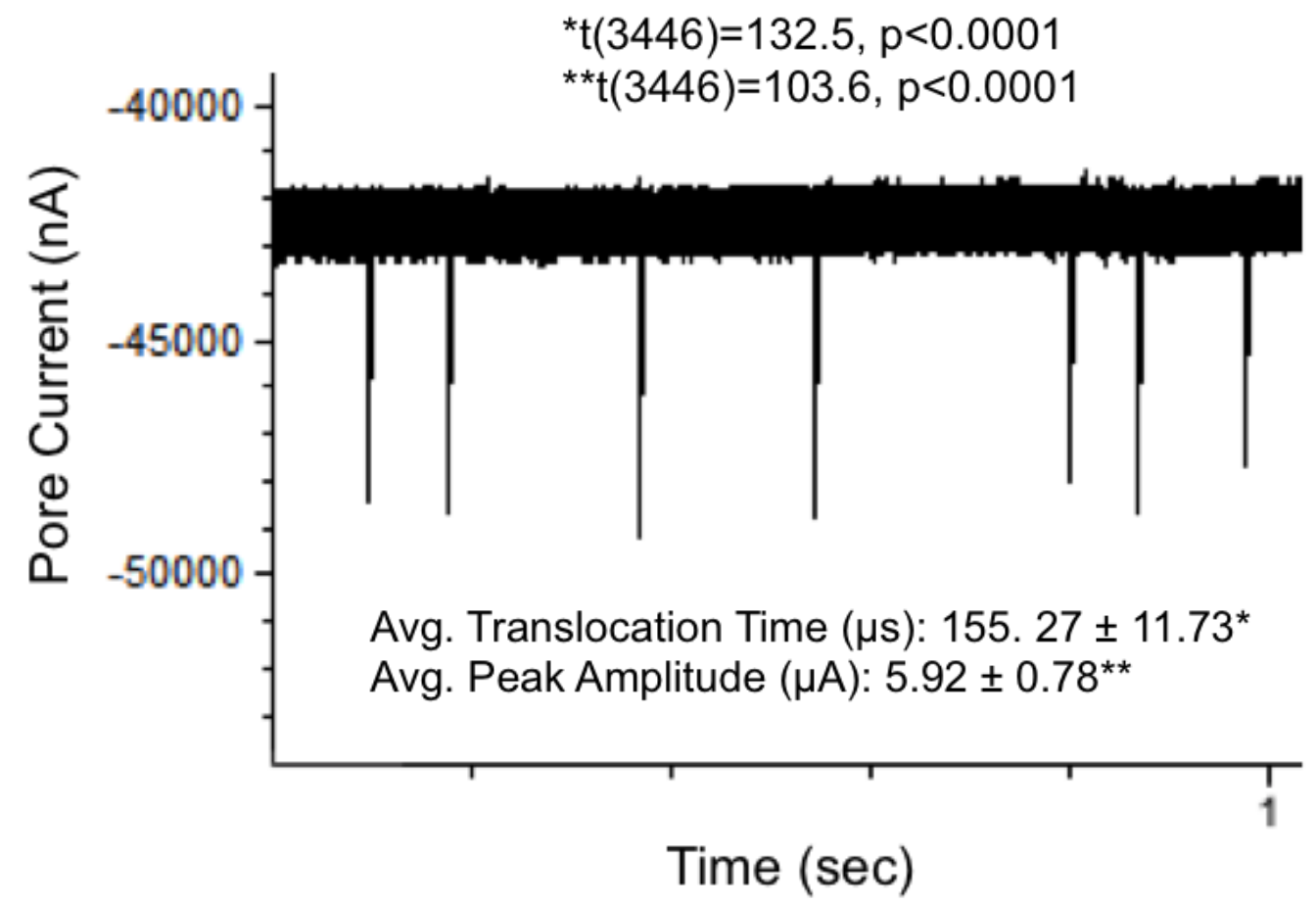

(A)

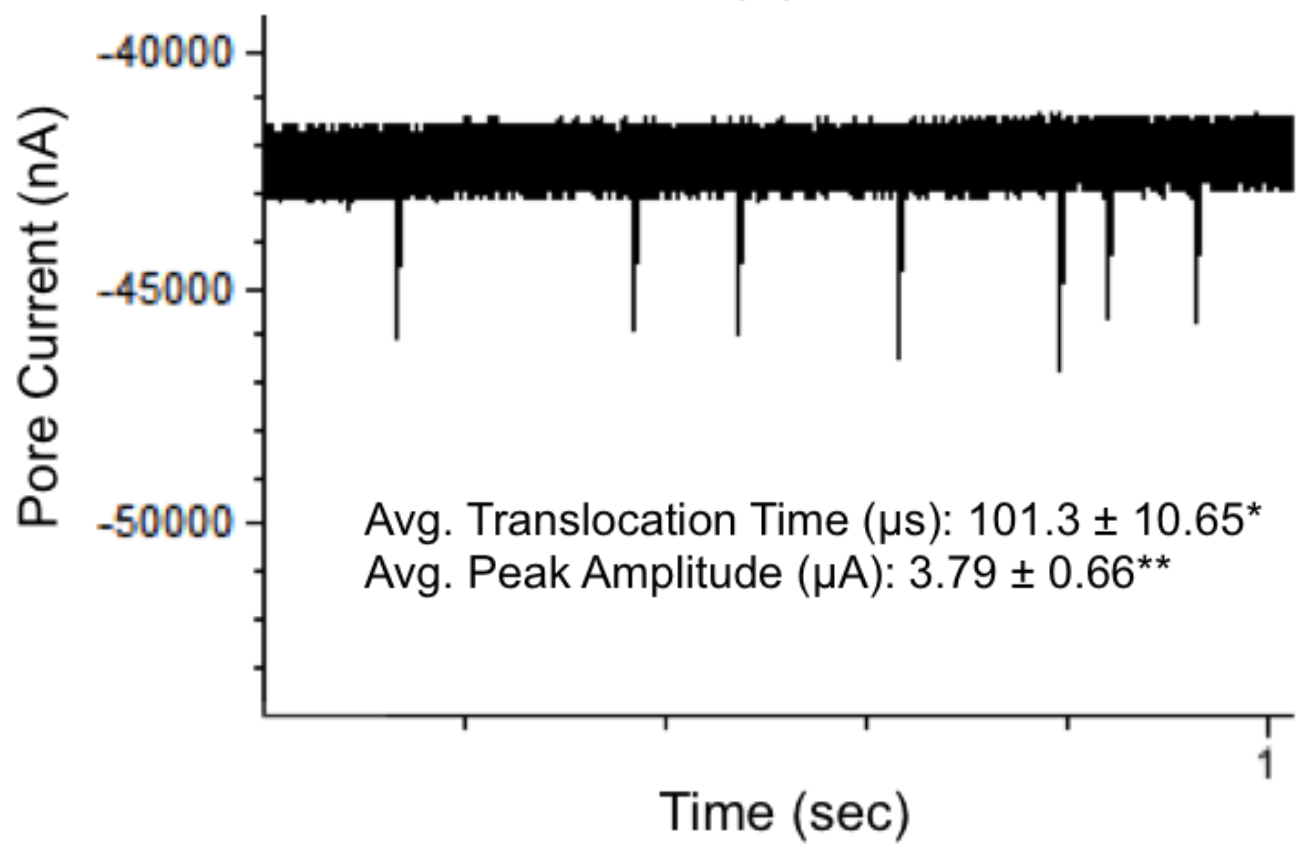

(B)

Figure S1. This data is from another run and it shows similarity to Fig 4. The temporal trace of ionic current shows pulses from the translocations of (A) NMTCs and (B) MTCs through the micropore run separately. 


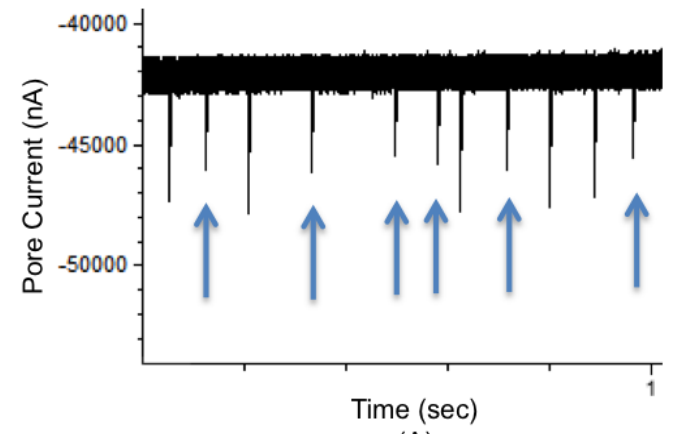

(A)

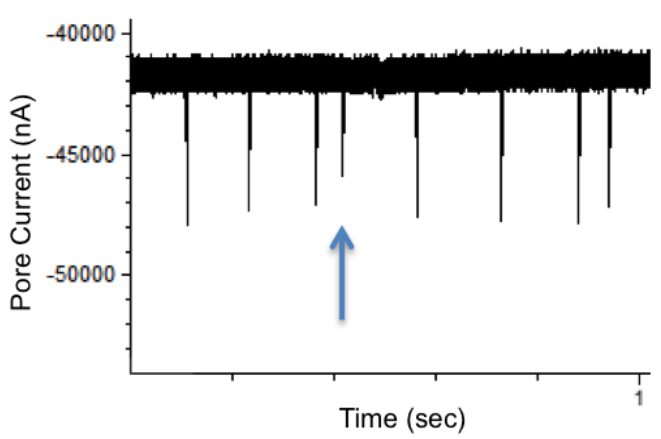

(B)

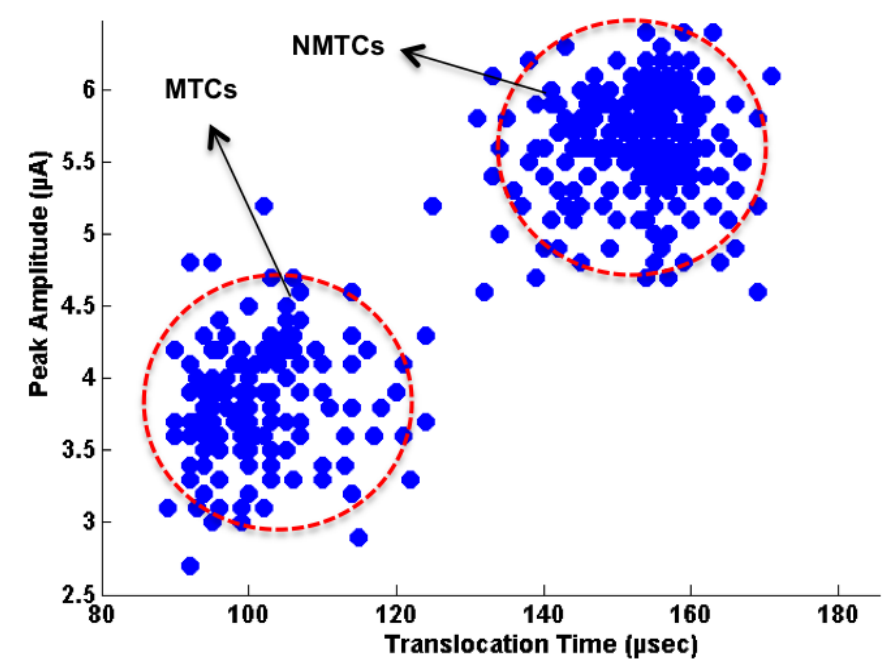

(C)

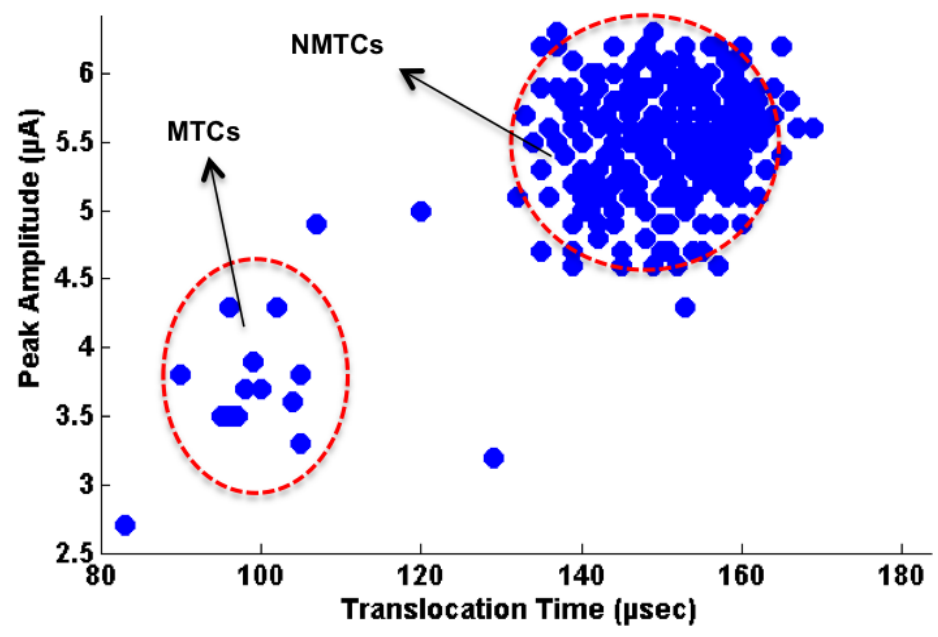

(D)

Figure S2. This data is in support of Fig. 6. This is from another run. It shows translocation of mixtures of NMTCs and MTCs through the micropore at 1:1 and 1:10 ratio. Temporal trace of the ionic current shows pulses from the translocations of MTCs and NMTCs in (A) 1:1 ratio (Mixture 1) and (B) 1:10 ratio (Mixture 2). Arrows indicate MTCs. (C) and (D) show the scatter plot of pulse attributes from mixed cell suspensions of MTCs:NMTCs at 1:1 ratio and 1:10 ratio, respectively. In both (C) \& (D), population of MTCs and NMTCs obtained from pulse attributes is inline with the actual ratio of MTCs and NMTCs in the mixed suspension. 


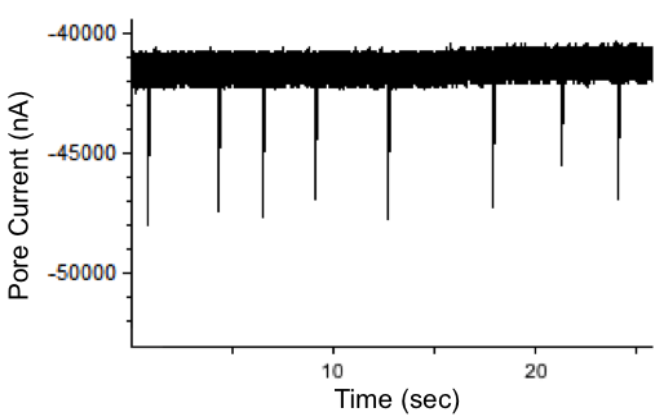

(A)

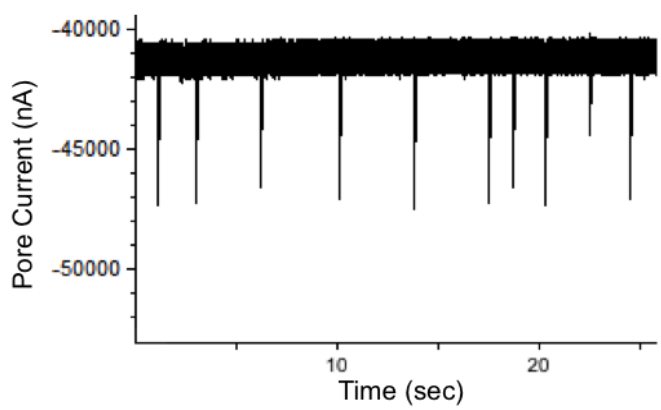

(B)

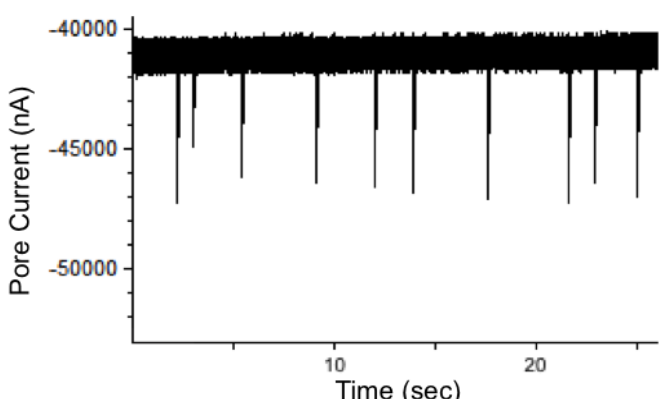

(C)

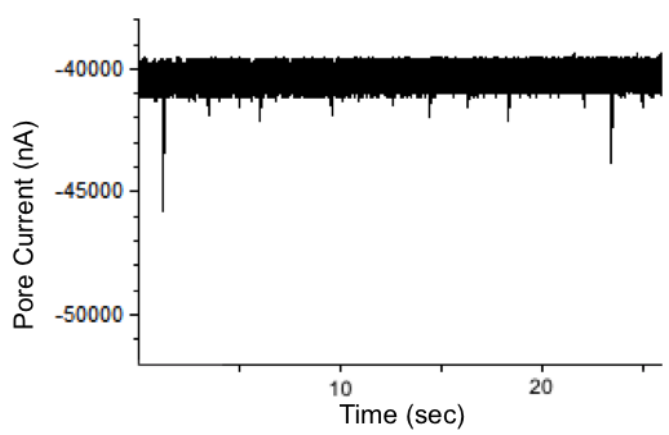

$(\mathrm{G})$

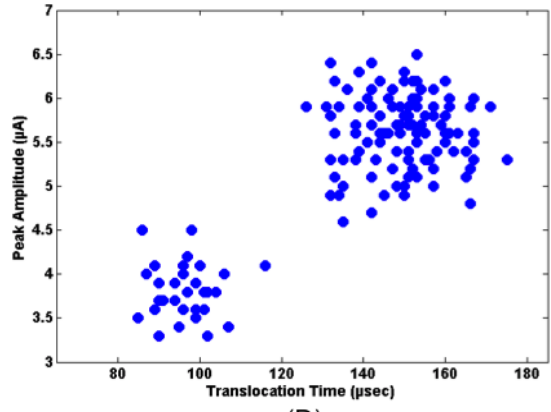

(D)

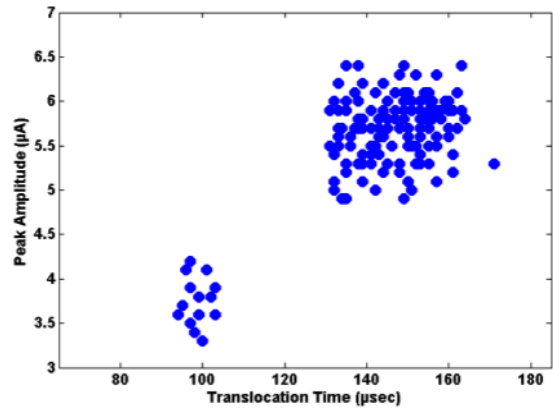

(E)

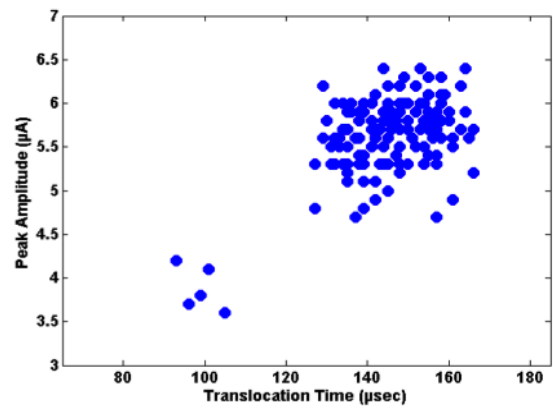

$(\mathrm{F})$

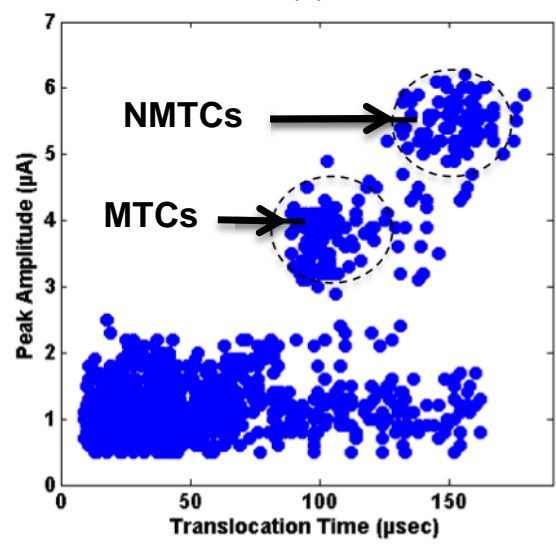

$(\mathrm{H})$

Figure S3. The data supporting Fig. 7. This is from another run. Translocation of mixture of NMTCs and MTCs through the micropore at (A) 1:25, (B) 1:50 and (C) 1:100 ratio. (D), (E) and (F) are their corresponding scatter plots, respectively. Each mixture is $10 \mathrm{ml}$ and contains $400(\mathrm{~A}), 200(\mathrm{~B})$ and $100(\mathrm{C})$ NMTCs with 10,000 NMTCs. $(G)$ and $(H)$ show the temporal trace of the ionic current and scatter plot for the mixture of 5000 cells of endothelial cells and stromal fibroblasts each and 2000 cells of NMTCs and MTCs each. 